$$
\begin{aligned}
& \text { N71-20322 } \\
& \text { NASACR-117036 }
\end{aligned}
$$

LOST CITY METEORITE - ITS RECOVERY AND A COMPARISON WITH OTHER FIREBALLS

R. E. McCrosky, A. Posen, G. Schwartz, and C. -Y. Shao

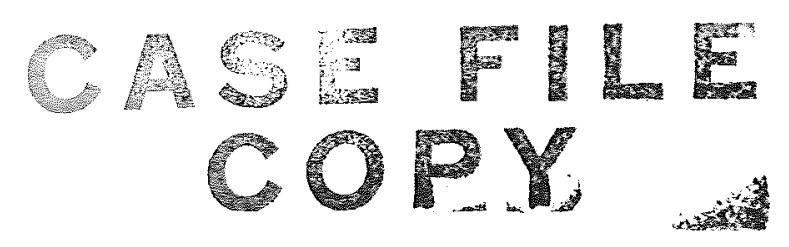

October 1970

Revised February 1971

Smithsonian Institution

Astrophysical Observatory

Cambridge, Massachusetts 02138 
Lost City Meteorite - Its Recovery and a Comparison with Other Eireballs

R. E. McCrosky

Smithsonian Astrophysical Observatory and Harvard College Observatory, Cambridge, Massachusetts 02138

A. Posen

Smithsonian Astrophysical Observatory, Cambridge, Massachusetts 02138

G. Schwartz

Smithsonian Institution Meteorite Network,

Lincoln, Nebraska 68508

and

C. - Y. Shao

Smithsonian Astrophysical Observatory and Harvard College Observatory,

Cambridge, Massachusetts 02138

The Lost City meteorite was photographed in flight and recovered by the Prairie Network. Four fragments totaling $17 \mathrm{~kg}$ have been found.

Photometric and trajectory data for the Lost City meteor and for a subsequent meteor (No. 40617) with similar flight characteristics are used to establish a preliminary calibration of the mass scale of other fireballs. There is excellent agreement between the photometric masses derived from the most recent determination of the luminous efficiency of the meteoric process, and the dynamic masses determined from the trajectory and the shape of the recovered meteorite. It is unlikely that photometric masses are overestimated by as much as a factor of 8 for objects of the size and composition of Lost City.

Previous values for meteoroid bulk densities may have been seriously underestimated (by a factor of 5 or 8 ) if the flattened shape observed for Lost City and inferred from 40617 is common among meteoroids. 
An analysis of a third meteor, No.40503, is presented as a counterexample to the above argument. This meteor of 100 times the intensity of Lost City reached maximum light and then was extinguished nearly instantaneously. We recognize that this is a different kind of meteoroid but have no satisfactory explanation for its behavior.

The orbits of these meteors are similar to those of most other fireballs.

\section{THE LOST CITY METEOR}

The Lost City meteor occurred over northeastern Oklahoma at 2014 CST on January 3, 1970. The fireball at maximum light was comparable in brightness to the full moon. It was widely observed. Sonic booms were reported to have been heard over an area of about $1000 \mathrm{~km}^{2}$ between Tulsa and Tahlequah. Well-confirmed reports of the audio event cover an area of at least $300 \mathrm{~km}^{2}$, including Tahlequah, Waggoner, Inola, and Mazie, Oklahoma. This was variously described as like thunder, a single boom, or a series of consecutive sharp cracks.

The meteor was photographed from four Prairie Network stations in Hominy and Woodward, Oklahoma, and Pleasanton and Garden City, Kansas [McCrosky, 1970]. Compared to most Prairie Network meteors, Lost City is unusual. The initial velocity $(14.2 \mathrm{~km} / \mathrm{sec})$ is in the lowest $6 \%$ of our meteors. The initial height $(86 \mathrm{~km})$ is substantially greater than that of the average low-velocity meteor. The end velocity $(3.5 \mathrm{~km} / \mathrm{sec})$ and end height $(19 \mathrm{~km})$ are both record lows for Prairie Net meteors. The duration of the photographed event ( 9.0 sec) is the third longest in our collection. The light curve (intensity vs. time) is remarkably smooth. The few slight flares are apparently associated with the fragmentation of the meteoroid. Two small fragments that depart from the main trajectory are visible at altitudes of $30-40 \mathrm{~km}$. They disappear within a few tenths of a second.

During the last 1.5 sec of the trail, three separate fragments are visible on the Hominy photograph. The other, more distant stations either do not show the end portion of the trail or have insufficient resolution. The faintest 
trail (III) is well separated on the photograph from the brightest (I) for the last 1 sec of its trajectory and can be measured as a separate object. At its end height $(24 \mathrm{~km})$, the velocity is $4 \mathrm{~km} / \mathrm{sec}$. The trail of intermediate brightness (II) is nearly superimposed on the brightest trail and is recognizable only because of its slight differential deceleration with respect to $I$. No recognizable trace of II occurs during the last $0.5 \mathrm{sec}$ of $I$.

Trail II is about one magnitude fainter than trail I, and trail III is very much fainter - about four magnitudes - than the others. Thus, both the brightness and the duration suggest that the fragments consist of two large pieces of detectably different but comparable size and one very much smaller piece. In addition, trails of other smaller pieces could be lost in the bright images of the main trails.

\section{IMPACT PREDICTIONS}

Measurements of the meteor photographs [McCrosky and Posen, 1968] give relative distances, $D_{i}$, along the trajectory line for each $i^{\text {th }}$ time interval defined by the chopping shutter. Experience has shown that the measures can usually be well represented by the expression

$$
D=a+b t+c e^{k t}
$$

The terminal parts of the trail for low-altitude objects like Lost City can be described by the simpler, constant-deceleration equation

$$
\mathrm{D}=\mathrm{a}+\mathrm{bt}+\mathrm{ct} \mathrm{t}^{2}
$$

In either case, the constants ( $a, b, c$, and $k$ ) are determined by a least-squares fit over a trajectory arc of $1--2-s e c$ duration. The velocity and deceleration are derived from equation (1) or (2) by differentiation.

The drag equation is

$$
m \dot{V}=-\frac{C_{D} S \rho V^{2}}{2}
$$


Where $S$ is the projected frontal area of the body, $m$ is the body mass, $V$ is the velocity, $C_{D}$ is the drag coefficient, and $p$ is the atmospheric density, or

$$
v \equiv \frac{m}{C_{D} S}=-\frac{\rho V^{2}}{2 \dot{V}}
$$

where $v$ is the ballistic coefficient. The right-hand side is known from the trajectory and the atmosphere. For trail I of Lost City, we find $v=22 \mathrm{~g} / \mathrm{cm}^{2}$ at a point near the end of the luminous trail.

The impact point was predicted by using the known position, velocity (including direction), and ballistic coefficient and by performing a numerical integration of the complete drag equation containing gravity and wind effects in addition to the body force. We used a standard atmosphere for the air density and took the winds aloft from the radiosonde flown from Oklahoma City at $1800 \mathrm{CST}, 2 \mathrm{hr}$ before the meteor. The wind was measured to $15 \mathrm{~km}$. The wind direction at all altitudes was $280^{\circ} \pm 10$, and the speed ranged from $5 \mathrm{~m} / \mathrm{sec}$ at the surface to $65 \mathrm{~m} / \mathrm{sec}$ at $8 \mathrm{~km}$.

Since we had no prior knowledge of the body shape, we assumed it to be spherical and of meteoritic density. In the terminal phases of its luminous flight, the ballistic behaviox of Lost City was like that of a $1.2-\mathrm{kg}$ sphere of meteoritic material. We assumed that the ballistic coefficient derived from the observations and the drag equation was the appropriate value to use in further integrations of the same equation. Thus, the integration was performed with a drag coefficient appropriate for a sphere, i.e., $C_{D}=0.92$ for the extreme supersonic-flow regime and dependent on Mach number, Ma, and on Reynolds number for $\mathrm{Ma} \leq 3$.

With the meteorite now in hand, we cannot improve on the predicted impact. The actual drag force as a function of time depends on complex and unknown variations in the drag coefficient and body orientation. These variations arise from changes in the direction of the drag force that occur, for example, whenever the wind field changes in direction or magnitude. The 
air stream will then be acting on a new surface (with a different ballistic coefficient) until such time as the meteorite can be reoriented to a stable flight position.

We can assess the degree of validity of our method of impact prediction directly only when we have made a recovery or indirectly by the following arguments. Consider, for descriptive purposes, the dark-flight trajectory to be roughly approximated by two independent sections. First, the body continues in its initial direction until decelerated to $\mathrm{Ma} \approx 1$. In the second section, the body is in vertical free fall except for any displacements by wind. For trajectories with moderate to large zenith angles of the flight path, say $z>30^{\circ}$, most of the horizontal displacement of the impact point from the last observed point will occur during the first section. So long as the ballistic coefficient does not vary appreciably during this portion, the spherical-body approximation is valid. The specific assumptions for the impact prediction are that (a) the meteorite does not ablate or fragment after the end of the Iuminous trajectory; (b) the body, if highly asymmetrical, has reached a stable orientation by the end of the luminous trajectory and does not tumble during dark flight; and (c) the variations in the drag coefficient with Mach and Reynolds numbers are proportional to those of a sphere, i.e., $C_{D}$ (body)/C $C_{D}$ (sphere) = constant. Condition (a) is probably met by most meteorites. The body has already passed through peak dynamic load, and the heating rate at $v<4 \mathrm{~km} / \mathrm{sec}$ must be very low. Condition (b) might fail if major fragmentation occurs near the end of the trajectory, permitting new torques to be applied to the body. Whether or not the body will quickly reach a stable position would depend on the details of the shape. Condition (c) is subject to experimental verification and can be attempted on a Lost City model.

Winds can displace the impact point of spheres of $1-10 \mathrm{~kg}$ by several kilometers. We have no more than intuition to guide us in estimating the errors arising from unknown shape in the second section of the extrapolated trajectory. Errors of $50 \%$ in the wind displacement seem unlikely for bodies of 'reasonable' shape. However, a flat plate in a constant-velocity windfield 
will assume a nonzero angle of attack with respect to the vertical and be subjected to a lift force that cannot be included in our computations.

Lift or other forces normal to the drag force may well occur for any nonsymmetrical body during any portion of the trajectory. The result of such forces is observed in the separation of the fragments in the Lost City photograph. When the observed meteor trail is artificially 'straightened' by removing the effects of gravity and atmospheric refraction (see McCrosky and Posen [1968] for discussion of these procedures), we find that trail III continues in the original flight direction and that trails I and II curve to the north. Trail $I$ has changed direction by about $1^{\circ}$ at the end. This is a minimum displacement in that we view only that component in the plane perpendicular to the line of sight. Figure l shows the computed submete orite ground path during the final portion of the dark-flight trajectory for the fragment responsible for trail I. The observed ballistic coefficient was used. The impact point for the zero-wind case is shown for comparison. A correction for the $1^{\circ}$ departure from the original direction has been applied in both cases.

An attempt was made to predict an impact from trail II. The measurements were extremely difficult, and poor, and we had very little confidence in them. A ballistic coefficient of $22 \mathrm{~g} / \mathrm{cm}^{2}$ was found, but the probable error could have been one-half of this. The velocity at the end of the trajectory is not well determined. Impact solutions for various ballistic coefficients and terminal velocities that might be reasonable cover a ground area of many hundreds of acres to the west of the trail I impact point.

Trail III could be measured with some precision, but given the low ballistic coefficient of less than $10 \mathrm{~g} / \mathrm{cm}^{2}$, we had very little confidence in the impact point (see McCrosky and Ceplecha [1969] for a discussion of impact errors as a function of body size). Our computed impact area for trail III was about $2 \mathrm{~km}$ to the northwest of that for trail I.

Figuxe 1 demonstrates that wind corrections are not negligible. The I ost City meteorite had particulaxly large displacements caused by winds of 
nearly jet-stream force. This disadvantage was at least partially counterbalanced by having the wind direction nearly the same as the flight direction. of the meteor. This circumstance is advantageous because errors in the ballistic coefficient tend to produce compensating errors in the impact point. For example, if too small a ballistic coefficient is used, the hypothetical meteorite would decelerate too rapidly during the first portion of the trajectory and would, in the absence of winds, fall short of the actual body. But during the second section when winds are the major agent, the smaller body will be given a greater displacement and thus tend to catch up with the real object by the time impact occurs.

These arguments also permitted us to make some generalized estimates on the location of fragments for which we had no trajectory data. We felt that smaller bodies could not proceed as far south as the largest piece during the high-velocity portion of its flight. Since the winds were almost due west, with only a.slight southerly component, we expected smaller pieces to lie north of the main fragment. Our computations further indicate that these smaller fragments should remain west of the largest one unless they were smaller than $1 \mathrm{~g}$.

\section{RECOVERY}

Within 4 days of the event, an impact area east of Lost City, Oklahoma, was predicted from the data. A snowfall of 7-9 in. occurred the day after the fall and closed most of the side roads until January 9. On the afternoon of that day, one of us (GS) recovered a $9.83-\mathrm{kg}$ meteorite on the surface of a dirt road. We currently believe this to be either the main mass or one of two primary pieces of the meteorite fall. It has a complete fusion crust. The leading surface is well defined by flow lines and is notably smoother than the aft surface. There was no obvious crater in the immediate vicinity of the recovery point. Snow prevented any careful inspection of the area at the time. Later, the melting snow produced a thick layer of mud, and traffic would probably have obliterated any crater shallower than about $10 \mathrm{~cm}$. No crater was found in the adjoining roadside. We believe the road was hard 
packed and dry at the time of fall. Perhaps cratering was negligible. However, we also suppose it possible that the meteorite landed elsewhere and was transported to the recovery site by means or persons unknown.

Lost City is in the foothills of the Ozarks, a region specifically excluded in the design of the Network because of the poor terrain for meteorite recovery. Scrub pine and oak forests are common, and the chance of recovery in these is negligibly small. However, the immediate surroundings of the predicted impact point consist equally of flat pasture land and woods partially cleared by grazing. Had the road not been there and had we initiated our normal search patterns about the predicted impact point, we would have had to search less than $0.8 \mathrm{~km}^{2}$ before reaching the actual recovery point. We believe that the existing snow and the snow that followed would have delayed recovery for about a month. Many radioactivity measures would have been seriously hampered.

More good fortune befell us with the geometry of the meteorite. This oriented stone should be of more than average value for ballistic and ablation studies. We were probably only a few hours too late in our recovery to meet the requirements of those biochemists with an interest in organic compounds in meteorites. Judging from the footprints around the meteorite and the stained snow immediately adjacent to it, we believe this object to have been seriously contaminated by a dog or some othe $r$ canine.

The point of recovery of fragment $l$ is labeled 'l' in Figure 2. Subsequent finds are numbered in chronological order of their recovery. Point 1 is about $700 \mathrm{~m}$ from the predicted impact point, I. Impact II, as noted previously, is very poorly determined. Its proximity to ' 1 ' is probably not significant. The distance of the Network stations from the recovery point exceeds that for any other meteorite within the Network. We could have reasonably expected a larger impact error for this case. In retrospect, we can understand the error component perpendicular to the flight path if the lateral force was maintained during dark flight. A negative lift force, which would not be apparent on the Hominy photograph, would be required to account for the error along the flight path. We think it more likely that the se errors are inherent in our prediction procedures or in the difference in the wind fields at Oklahoma City and Lost City. 
A measurement of the main mass gives $\mathrm{m} / \mathrm{s}=26 \mathrm{~g} / \mathrm{cm}^{2}$. If our measurements are without error and if the main mass was responsible for trail I, then the drag coefficient for this body in supersonic flow would be $C_{D} \approx 1.2$. Wind-tunnel measurements of a model are required to determine the actual drag coefficient. Any reasonable discrepancy between the prediction and measurement can be absorbed by a change in the assumed shape, but it may also indicate that our association of the meteorite with the brightest trail is in error.

At this time, we accept the correlation between the main mass and trail I because of the apparently valid impact prediction and of the fair agreement between the ballistic coefficient measured in flight and that expected for the meteorite. The most compelling evidence to the contrary would be the recovery of a still larger fragment, which would have to be correlated with the brightest trail regardless of its place of impact.

A second, small fragment (272 g) was found on January 17 by Philip Halpain while he was walking through his pasture. Fragment 2 is a highly flattened piece of roughly rectangular shape. The fusion crust is complete. The meteorite landed with its flat surface down and its heavy end in the forward direction. It was buried in the sod to its own depth. We propose the name 'Lost City Meteorite - Halpain Fragment' for this specimen in acknowledgment of Mr. Halpain's discovery and his thoughtful generosity that permitted an early analysis of the fragment for short-lived isotopes.

Our initial search was in the region to the northwest of no. 1, where we expected smaller material to land. The area was mostly forest with considerable undergrowth. The chances of recovery here are exceedingly small. The area of recovery of the third piece was to the east of our primary search region. We searched this area sooner than originally planned after interviewing Mr. Rufus Yaeger, a local resident who, by the greatest good fortune, was able to report a unique aspect of the meteor event. He lives about $600 \mathrm{~m}$ north of point 3 (Figure 2). He was unaware of the fireball but happened to leave his house during the $90-$ sec period between the end of the photographed 
trajectory and impact. He was outside before the first sonic booms arrived. They drew his attention to the western sky, where he believes he saw three red lights. While his detailed observations of the phenomena are inconsistent with what we know of the trajectory, there is not much doubt that he was viewing, for perhaps $10 \mathrm{sec}$, more than one glowing meteorite. At the time, he was under the impression that he was witnessing an aircraft in distress. In this excitement, it can be expected that his observations would not be perfect. He was certain, however, that after the visual event was over, he heard 'a thud, like something heavy hitting the ground' to the south of him. The main impact was a mile away and the small Halpain fragment a half mile away. It seemed to us unlikely that he could have heard either of these.

Fragment $3(6.6 \mathrm{~kg})$ was recovered on February 2 by Schwartz, Ivan Burr, James Sohl, and J. T. Williams, all of SAO, in the course of a systematic search. It was found in the bottom of a hole $30 \mathrm{~cm}$ deep in a rough pasture. The hole diameter, $22 \mathrm{~cm}$, was comparable to that of the meteorite. Many other equally suspicious depressions can be found in the pasture. These are caused by old animal burrows collapsed by cattle or are entrances to these burrows. A subsequent excavation around the crater area failed to show a burrow associated with the crater.

The meteorite crater was thought to be unusual but not sufficiently so to photograph it before the details were destroyed by our initial excavation. It was noted, however, that about $5 \mathrm{~cm}$ of dirt covered the meteorite, the top of the dirt layer being about $15 \mathrm{~cm}$ below the surface. Two small cracks extended out from the crater wall about $2 \mathrm{~cm}$, one to the east and the other to the southeast. At the end of each crack and parallel to the crater edge, there was some loose dirt about $2 \mathrm{~cm}$ high and $10-15 \mathrm{~cm}$ long. The meteorite has a complete fusion crust and a suggestion of flow lines. The smoother ablation surface, which is characteristic of the leading surface of fragment 1 , occurs as a small area of this fragment, indicating that it may not have flown in a maximum-drag orientation.

Fragment $4(640 \mathrm{~g})$ was recovered by Sohl, in the company of Schwartz and Burr, on May 4. This piece also has a completefusion crust. The meteorite was lying on the surface. No crater was found. 
On the face of it, it is difficult to see how this smaller body and the third fragment could have landed to the east of the main mass, i.e., farthex along the trajectory, unless they had substantial positive lift with respect to the main body. If fragment 3 is the object associated with trail II, then our impact error exceeds $2 \mathrm{~km}$. With regard to future impact predictions, these fragments cause us as much concern as the first piece gives us confidence.

Approximately $2.6 \mathrm{~km}^{2}$ was searched with care during the course of 500 man-hours of walking. An additional $0.8 \mathrm{~km}^{2}$ of difficult terrain was covered, with greatly reduced efficiency, during 100 man-hours of search. We concluded our recovery effort on December 6, 1970.

\section{PHOTOMETRIC AND DYNAMIC MASSES OF THREE METEORS}

A. Photometry

Photometric methods used for Prairie Network meteors have been described in detail elsewhere [McCrosky and Posen, 1968]. Briefly, comparison of the density of the meteor image is made with that of the trails of stars of known brightness on the meteor film. Appropriate corrections are made for the relative trailing velocity of meteor and star, color differences of the stars, and certain optical and photographic effects. The result is expressed in apparent stellar magnitude, $M_{\text {app }}$. Thus, a 0 -mag meteor would produce the same image as a 0 -mag AO star if the star image were moved across the film plane at the meteor rate. Absolute meteor magnitudes, $M$, are apparent magnitudes adjusted to a standard distance of $100 \mathrm{~km}$. Intensity is related to magnitude by

$$
M=-2.5 \log I .
$$

A comment on the special nature of the Prairie Network photometric results is pertinent. Most astronomical magnitude scales, including most meteor astronomy scales, are specified for a limited region of the spectrum. The most common meteor systems are the so-called photographic (pg) and 
visual $(v)$. In the photographic system, the upper wavelength limit of sensi... tivity is that of a blue-sensitive emulsion, $\lambda \lesssim 5000 \AA$, and the lower limit is that determined by the absorption of the atmosphere or the optics, $\lambda \geq 3500-$ $3800 \AA$. The visual magnitude scale can be either what the name implies (i. e., based on the response curve of the eye) or based on a photographic or photoelectric recording system with a maximum response at $\lambda \approx 5400 \AA$, where the eye is most sensitive. Sources with different spectral distributions, of course, produce different responses in the two systems. A zero point for any magnitude scale is established by assigning the same numerical magnitude to a star of spectral class A0. For other objects, the difference in the magnitudes defines a color index, C.I. $=M_{p g}-M_{v}$.

Prairie Network data are recorded on panchromatic film, and thus our scale corresponds to neither of the usual ones. (The choice of film is dictated in part by compelling economic reasons - most of our film has been obtained without cost from Defense Department surplus.) We have found the empirical relationships $M_{\text {pan }}=M_{v}+0.3$ (C.I.) for stars earlier than spectral class K2 (i.e., for all but the very red stars). We will show later that $M_{p g} \approx M_{\text {pan }}$ for meteors. Magnitudes quoted hereafter without subscripts are absolute, panchromatic magnitudes.

Both the meteor's luminosity and its ballistic coefficient are, in some way, related to its mass. Most attempts to provide knowledge about the physics of the meteor process or about the structure of meteoroids rely on comparison of these nearly independent indicators of mass. For bodies of unknown shape and density, the area factor in equation (3) can be expressed as

$$
S=A m^{2 / 3} p_{m}^{-2 / 3}
$$

where $\rho_{m}$ is the bulk density and $A$ is a dimensionless shape factor. We then define the dynamic mass $m$ as

$$
m_{d}^{1 / 3}=-\frac{C_{D} A V^{2} \rho}{2 \dot{V} \rho_{m}^{2 / 3}}
$$


Almost all meteor spectra show that meteor luminosity is due primarily to excited meteor atoms. This is true for meteors more than 100 times fainter than Lost City. It is also true for a meteor of - $18 \mathrm{mag}$ - more than 100 times brighter than Lost City - observed by Ceplecha. Details of the spectrum are unpublished, but a brief summary has been given by McCrosky and Ceplecha [1970]. Many spectra due to cometary meteoroids have been published. There are, of course, no spectra of meteors known to be produced by stony meteoritic material. We assume the two types of spectra have similar characteristics. To the extent that the luminosity is produced by the meteor atoms, the observed intensity must be related to the rate of vaporization of the meteoroid. For meteors considerably fainter than Lost City $(M \sim-5)$, the intensity, $I$, can be represented, to within the limits of the observations, by

$$
I=-\frac{T}{2} V^{2} \frac{d m}{d t}
$$

where $\tau$ is the luminous efficiency factor. The photometric mass at any time, $t$, of the trajectory is given as

$$
m_{p}=2 \int_{t}^{t} \frac{I}{\tau V^{2}} d t
$$

In the following, we will compare the photometric mass with the dynamic mass. Anticipating our result, we will find that the currently available data are sufficient to place reasonable limits on a scale-factor correction to our chosen luminosity law. We cannot determine variations of the scale factor along the trajectory. The correction factor may encompass (a) real differences in the luminous efficiency factor between bright and faint meteors; (b) gas-cap radiation, which we explicitly ignore because of its apparently minor role in meteor spectra; and (c) errors in measurement.

Our purpose, then, is to provide an improved mass calibration for other fireballs: We hope that studies of cosmic-ray effects will eventually provide limits on an important initial condition - the preatmospheric mass - that has 
heretofore been an unknown, to be determined from the meteor observations. With the added constraint on initial mass, further improvements in the calibration will probably be possible.

We include in this report a second Prairie Net meteor, 40617, that occurred after Lost City on January 31, 1970. The meteor was three magnitudes fainter than Lost City and occurred at $3 \mathrm{a} . \mathrm{m}$. It did not attract any local attention; we learned of the event during routine inspection of our film. We estimate the terminal mass to be substantially less than that of Lost City. A search, in excellent terrain in Nebraska, more extensive than that for Lost City has yet to yield a meteorite. Otherwise, the similarities between the two events are remarkable. These include initial and final velocities, the amount of atmosphere penetrated, the duration, and the occurrence of multiple fragments that persist for an appreciable fraction of the trajectory. Both meteors were better observed than any previous deep-penetrating Prairie Net objects.

We will also discuss a third Prairie Net meteor, 40503, as a counterexample to demonstrate that an unconditional application of a calibration derived from Lost City and 40617 is probably unwarranted. Meteor 40503 provides strong evidence that not all bright fireballs are structurally similar to Lost City. It was much brighter than Lost City and produced sonic booms of at least the same intensity. We will contend that it could not have produced a meteorite in excess of a few tens of grams.

Lost City. Meteors of high apparent intensity greatly exceed the dynamic range of our optical systems. Photographs from distant stations are commonly used for photometry of brighter meteors. The Lost City photometry was accomplished primarily with the films from Pleasanton and Woodward. The overexposed Hominy trail provided data for only the beginning and ends of the meteor. Sky conditions were excellent at all stations. Where photometry is possible from two stations, the agreement is better than 0.5 mag. 
In the following, we use a smooth curve (Figure 3) drawn through the observed points. The position of a small but distinct flare visible on more than one film is shown schematically. The intensities of the three fragments have been summed for the light curve of the final $1.5 \mathrm{sec}$.

Meteor 40617. This meteor was photographed from 10 stations with excellent sky conditions. The faint limiting magnitude and the easy resolution of the three terminal fragments are the result of the small range $(R=80 \mathrm{~km})$ of the meteor from the Republican City, Nebraska, station. The brighter portions of the trail slightly exceed the dynamic range for photometry from this station, and that part of the light curve (Figure 3) was determined from the next nearest station, at Steinauer, Nebraska. The flares at $t=4.95$ and $t=6.3 \mathrm{sec}$ have been confirmed by relative photometry from three stations. The photometry for this fainter object is superior to that for Lost City.

Meteor 40503. This meteor was photographed from nine stations. The bright flares of the meteor exceed the dynamic range at all stations. The entire film from the nearest station is partially fogged by the meteor. Individual clouds are not illuminated, and we believe the fogging is due primarily to scattered light in slightly hazy atmosphere. The two terminal flares on this film show image reversal in the core of the image, a unique characteristic of this Prairie Net fireball, and one that we hoped would provide a calibration for the meteor. Image reversal occurs when an intense, short-duration pulse of light is applied to discrete image centers in the emulsion. We attempted, without success, to produce image reversal of the sun's image. The camera was pointed at the sun, and the film transported through the focal plane at a speed corresponding to the angular rate of the meteor. At the focal plane, a diaphragm of about 10 times the diameter of the sun's image was used to reduce the sky fog. In addition, it was necessary to use a neutral filter of density $=1$ to limit the light. The same lens, roll of film, and development procedure used for the meteor photograph were used in these tests. 
The angular diameter of the sun is distinctly larger than that of the meteor, and some correction is required to account for the difference in specific intensity seen by the film in the two cases before limits can be placed on the meteor intensity. We will first take the minimum angular size of the meteor, $D_{M}=4: 5$ (arcmin) to be equal to that of the reversed core of the image. It is unimportant whether this quantity represents the actual meteor size or the image size (meteor size increased by aberration) since in either case it is this area of the film over which the specific intensity was great enough to cause reversal. The panchromatic magnitude of the sun is $M=-26.7$. Allowing $0.3 \mathrm{mag}$ for atmospheric absorption and $2.5 \mathrm{mag}$ for the filter, the sun appears to the camera as a source of $\mathrm{M}_{\mathrm{V}}=-23.9$. A fraction of this source, given by $\left(D_{M} / D_{\odot}\right)^{2}$ where $D_{\odot}=32^{\prime}$, the sun's diameter, did not produce reversal, and we conclude that the apparent magnitude of the meteor at its brightest point must then be

$$
M_{\text {app }}<-23.9-5 \log D_{M} / D_{\odot}-2.5 \log 1.2=-19.8
$$

The radiation at the sun's center is 1.2 times its mean radiation. The final term in the equation corrects for the limb darkening.

The above limit is conservative since we have corrected for atmospheric absorption in the case of the sun but not for the meteor, which occurred in a less favorable sky. Furthermore, in reducing the sun's magnitude, we have used a minimal area of the sun, corresponding to the effective area of the meteor. In fact, the entire equatorial zone of the sun of width $4 ! 5$ might contribute to the reversal effect. In this second case, we can ignore limb darkening, and we have approximately

$$
M_{\text {app }}<-23.9-2.5 \log \left[\left(\frac{\pi D_{M}^{2}}{4}\right) /\left(D_{M} D_{\odot}\right)\right]=-21.6
$$

The range of the meteor at the flare was $R=53 \mathrm{~km}$. The correction to the standard range of $100 \mathrm{~km}$ is $\Delta M=-5 \log R / 100=1.4 \mathrm{mag}$, and the absolute magnitude of the flare, depending on which of the two models of the solar image is accepted, is 


$$
M<-18.4
$$

Or

$$
M<-20.2
$$

In the following, we take the round value of $M=-20$ for the brightest flare, noting that we do not claim an accuracy of better than 2 mag for this very difficult case. The remainder of the light curve shown in Figure 3 has been determined by standard procedures for portions with $M>-12$. Intermediate intensities, i. e., brighter than $M=-12$ but too faint to show reversal, were determined from two distant stations. The more distant of these, Woodward, Oklahoma, was partly cloudy. A comparison of the overlapping regions of the light curves from Woodward and Pleasanton suggested an additional atmospheric absorption correction of 0.1 mag/air mass for Woodward. The corrected magnitude of the brightest flare is again found to be $M \approx-20$. However, the total absorption correction for the final portion of the trail is nearly 10 mag. The agreement between our estimate of brightness of the first flare from the image reversal and its brightness from the Woodward data should not be given undue weight. Both determinations are uncertain.

A third method of crude photometry was attempted. Exposures of 1/15 sec to the daylight sky, with the sun at the film center, were made through filters of density $=2$ and density $=3$. The sky fog on the first of these closely matched that of the meteor film, while the sky fog on the second was markedly less. If the scattering properties of the atmosphere were the same for meteor and solar exposures - an admittedly weak assumption - the fog produced by an object with the light curve given in Figure 3 should equal that of the sun if exposed for $1 / 15 \mathrm{sec}$ with a filter of density $\approx 2.2$.

The photometric results are of low accuracy but are adequate to reach two conclusions about meteor 40503: (a) this is the brightest object observed in 6 years of operation, and (b) the integrated intensity of 40503 is of the order of 100 times that of Lost City. 
B. Photometric Mass

A debate has lasted for several decades over the appropriate expression of the luminosity law for meteors. A primary purpose of the Prairie Network was to place under observation objects of known density so that that quantity could be disengaged from the luminous efficiency when photometric and dynamic masses were available for the same object. A commonly used law for the luminous efficiency has been $\tau=\tau_{0} V$, where $\tau_{0}=10^{-19}$ when the intensities are on the photographic scale and in units defined by equation (5) and when the other quantities are cgs. This number was determined by artificialmeteor experiments and confirmed by unusual meteors that were presumed to have the density of asteroidal stone. A more complex relationship has recently been estimated [Ayers et al. , 1970] from a larger number of artificial iron meteoroids in the velocity range $8-16 \mathrm{~km} / \mathrm{sec}$. This new result is therefore particularly pertinent to low-velocity objects, such as Lost City. Although it may not be representative of the luminous efficiency of very large objects, we accept it, pro tempore, as the best present estimate. Our meteors produce substantial luminosity at $\mathrm{V}<8 \mathrm{~km} / \mathrm{sec}$. We have extended the experimental curve given by Ayers et al. to $6 \mathrm{~km} / \mathrm{sec}$ by a linear extrapolation of log t vs. $\log \mathrm{V}$. If the extrapolation is extended to still lower velocities, we would have to conclude that the meteor loses more mass per unit time at low velocity than at high. We have therefore arbitrarily set $\tau=$ constant and equal to its value at $V=6 \mathrm{~km} / \mathrm{sec}$ for $V<6 \mathrm{~km} / \mathrm{sec}$. This affects the initial photometric mass very little and makes a significant change only at the very end of the trajectory.

The meteor luminosity for low-velocity objects ( $\mathrm{V} \leq 20 \mathrm{~km} / \mathrm{sec}$ ) arises almost entirely from iron radiation. Other material except sodium is essentially inert. The analysis of Lost City [Clark et al., this issue] gives $27.6 \%$ iron by weight. Accordingly, we have used in our computations 0.28 times the value of $T$ determined from the artificial iron meteoroids.

Additional corrections to the Luminosity law are required because of the difference between the photographic pass band used in most of the artificial meteor observations and the panchromatic pass band used in the Prairie 
Network system. Ayers et al. find that $\log \tau_{p g}=\log \tau_{p a n}=0.2$. On the other hand, the resonance lines of sodium contribute to the panchromatic luminosity in natural meteors. The artificial meteors contained no sodium, and an additional correction should be applied to find a value, Tpan, appropriate for natural meteors. The two corrections are of the opposite sign. Given the uncertainty in the values of $T$ that will be derived from these meteors in the next sections, we will ignore this small net correction and use $\tau_{p g}=\tau_{\text {pan }}$. This is equivalent to the relationship $M_{p g}=M_{\text {pan }}$ given earlier.

C. Dynamic Mass

Lost City. The critical measurement for determination of dynamic mass is the deceleration. For very large bodies, this quantity is much smaller than its probable error near the beginning of the trajectory. Sometimes meaningful measures are possible only in the last third of the trajectory. In very bright meteors, the shutter breaks are often obscured by overexposure, and deceleration measures are impossible. This was apparently true for the Hominy photograph of Lost City, but we found that with a judicious recopying of the photograph under high contrast, the shutter breaks could be clearly identified along the entire trail. Decelerations were determined by the use of equation (1) for various 1.5- and 2-sec trajectory arcs. The deceleration at the center of an arc was used to determine the ballistic coefficient, $v$, of the meteoroid at that time. All solutions for $t<4$ sec showed internal probable errors of the deceleration comparable to the quantity itself, a not unexpected result for this massive body.

Eventually, we want to compare the dynamic mass as deduced from the ballistic coefficient with the photometric mass, which includes all the fragments. Accordingly, we have adjusted the observed ballistic coefficient to correspond to the quantity that would have been observed had no fragmentation occurred. For Lost City, where most of the mass appears to be about 
equally divided between the two bright trails, we have corrected $v$ by the factor $2^{1 / 3}$ for those portions of the trail after the fragmentation occurred. The time of breakup can be estimated to within about 0.5 sec by measuring the point of convergence of the fragments. This time is found to be near the major flare at $t=6.3 \mathrm{sec}$. We assume that the flare marks the time of the breakup precisely.

The ballistic coefficients, corrected for fragmentation when necessary, are plotted in Figure $4 \underline{a}$. The solid lines were fitted to the points by eye. In fact, the data are consistent with the dashed line, showing an abrupt change in $v$ at the time of breakup.

Meteor 40617. The deceleration measures for this meteor were superior to those for Lost City because of the smaller range and the ease of measurement of this fainter trail. Independent measures from the two nearest stations (Figure $4 \underline{b}$ ) show good agreement. The correction for multiple fragments is, however, more complex and thus subject to greater uncertainty. A breakup at $t=4.95 \mathrm{sec}$ is marked by the major flare and is also consistent with the point of trail convergence. The two trails have similar but detectably different intensities, and we have assumed that a correction factor of $2^{1 / 3}$ is appropriate until the time $t=6.6 \mathrm{sec}$, when the fainter fragment again breaks up. The observed $v$ for each of the final three pieces are nearly identical, and a correction of $3^{1 / 3}$ is used for $t>6.6$ sec. Our model is of course inconsistent at $t=6.6$ sec. Three equal pieces cannot be produced by breaking one of two equal pieces. However, an alternate model of an initial fragmentation into masses of ratio $2: 1$ and a subsequent division of the larger piece is clearly inconsistent with the relative intensities of the initial fragments. The difference in the correction factor between the two models is almost inconsequential, anyway.

In Figure $4 \underline{b}$ the dashed curve again represents our estimate of the variation of the corrected ballistic coefficient. The increase of $v$ in the vicinity of the major flares in both meteors is of course partly an artifact introduced by our correction for multiple fragments, but the discontinuities, as drawn, are about $50 \%$ larger than the correction factors. Changes in either shape or drag coeficient after breakup may be responsible. 
Meteor 40503. Trajectory measures for this meteor are impossible from the nearest station for all but a few beginning dashes. At distant sta tions, the final portion of the meteor is either off field or the data are degraded by the great range. Only the second nearest station gives any useful information on deceleration. The shutter breaks are not visible in any of our measuring engines, but they can be clearly detected in densitometer tracings made with the slit scanning in the direction of the meteor's motion. The positions of the breaks relative to a recognizable point early in the trail were read from tracings. The measures were consistent with those made on a very high-contrast copy of the original film. A single deceleration solution for the $\operatorname{arc}(4.5 \leq t(\mathrm{sec}) \leq 5.5)$ was obtained. We find $v=66 \pm 3 \mathrm{~g} / \mathrm{cm}^{2}$ at $\mathrm{t}=4.75$. The quoted uncertainty is the rms error derived from the rms error of the deceleration as determined in the leastsquares solution, but this formal error can be unrealistic. We are reluctant to accept a single determination without an independent confirmation from a separate film or an adjacent trajectory arc. Such confirmation is not available in this case, but we can add some credence to our result by a very unusual procedure forced on us by this unusual meteor.

Decelerations are second derivatives of observational data. They are often small quantities, and it is not unusual for the initial portion of a trajectory - or any portion of a poorly determined trajectory - to show formal errors of the deceleration comparable to the value itself. Velocities, on the other hand, are first derivatives of observations and are generally well determined.

The trajectory data for 40503 are plotted in Figure 5 in a fashion to demonstrate an astonishing proportionality relationship between this meteor and Lost City. The time scale for 40503 has been expanded by a factor of 1.5 and the velocity scale contracted by the same factor. The velocity and height curves are almost superimposed if an arbitrary I-sec shift of the time origin is applied. (The curves would, for the most part, be indistinguishable in the drawing if we had applied a 0.85 -sec shift.) The coincidence of the height curves results from a purely chance equality of the zenith angles of the radiants. The similarity of the velocity curves as plotted shows that any 
time-independent function of the meteors $\left(\mathrm{e} \cdot \mathrm{g} \cdot, \mathrm{V}^{2} / \dot{V}\right)$ is almost the same for any value of abscissa. Furthermore, since the absolute value of the height and therefore the atmospheric density also agree at any such point, we can conclude that $p V^{2} / 2 \dot{V}=v$ takes on about the same value for both meteors at corresponding times.

It is not our usual practice to determine ballistic coefficients by inspection of velocity curves, but, lacking well-determined deceleration data on 40503 and taking advantage of the fortuitous similarity with Lost City, we believe we have demonstrated that its dynamic mass is comparable to that of Lost City and that our single determination of $v=66 \mathrm{~g} / \mathrm{cm}^{2}$ is of the right order.

\section{INTERPRETATION}

We begin the analysis of these data by following the classical single-body theory that has given consistent results for other meteor data. Application of this theory requires an estimate of the terminal mass of the meteor - that part of the mass that has not vaporized - and, therefore, has not contributed to the light-producing process. The terminal mass, the $n$, is a correction to be added to the photometric mass. We will introduce simple variations in the constants $\tau$ and $C_{D} A$ (particularly for Lost City and 40617), either as suggested by the observations or as an attempt to find reasonable extremes of these constants. The implications for previous interpretations of fireball data will be discussed in Section 6.

We assume that Lost City and 40617 were homogeneous bodies and similar to the meteorite. We take it as self-evident that 40503 must differ from these two in some fundamental characteristic.

A. Terminal Mass.

The recovered mass of Lost City is $17 \mathrm{~kg}$. It is most unlikely that our recovery efficiency has been perfect. We suppose that small pieces remain but that they may be less than the present total. We noted earlier that 
another major fragment, comparable to the largest one recovered, may exist. A total ground mass of $20-30 \mathrm{~kg}$ appears to be a reasonable estimate. In the following, we have assumed a terminal mass of $25 \mathrm{~kg}$.

The terminal mass of 40617 is almost certainly less than that of Lost City. The value of $v$ of each of the three fragments at the end of their trajectories is $17.5 \mathrm{~g} / \mathrm{cm}^{2}$, about $2^{-1 / 3}$ of that of the main trail of Lost City. If each of the three pieces has the same value of $\mathrm{C}_{\mathrm{D}} \mathrm{A}$ derived for the main mass of Lost City, then the total terminal mass is $3 \times 1 / 2 \times 9.8 \mathrm{~kg} \approx 15 \mathrm{~kg}$.

A comparison of the intensity of the two meteors at a point near the end of their trajectories suggests that this is probably an upper limit. If the intensity is produced either by meteoric line radiation or by gas-cap radiation, then, to a good approximation

$$
\text { I } \propto S \rho
$$

for objects of the same velocity.

The heights of the main trail of Lost City and any of the three trails of 40617 when $\mathrm{V}=4.5 \mathrm{~km} / \mathrm{sec}$ are, respectively, $19.7 \mathrm{~km}$ and $25.9 \mathrm{~km}$. Atmospheric densities at these heights are $9.3 \times 10^{-5} \mathrm{~g} / \mathrm{cm}^{3}$ and $3.5 \times 10^{-5} \mathrm{~g} / \mathrm{cm}^{3}$. The main trail of Lost City is $M=-4.6$, and one trail of 40617 is $M=-2.0$. The ratio of the intensities is then, from equation (5), $I_{\text {L.C. }} / I_{40617}=11.0$. From equation (12) and the measured frontal area of fragment 1 , we find

$$
S_{40617}=\frac{9.3 \times 10^{-5}}{(11.0)\left(3.5 \times 10^{-5}\right)} S_{L . C .} \approx 90 \mathrm{~cm}^{2}
$$

Given the terminal value of $v$ and assuming $C_{D} \approx 1$, then the terminal mass is about $1.5 \mathrm{~kg} / \mathrm{fragment}$ or about $5 \mathrm{~kg}$ total. We take an average of these two, $10 \mathrm{~kg}$, as a first estimate of the terminal mass in the following. 
We stated earlier that we consider the terminal mass of 40503 to be very small. We reached this conclusion by comparing the luminosity of the meteor with that of Lost City at comparable points in the trajectory. At an altitude of $26 \mathrm{~km}, V_{\mathrm{L}} . \mathrm{C} . \approx 8 \mathrm{~km} / \mathrm{sec}$ and $M \approx-9$. Any sizable terminal fragment of 40503 at this altitude, which is just below the terminal flare, would have $\mathrm{V} \approx 12 \mathrm{~km} / \mathrm{sec}$. Yet despite the greater velocity, the intensity of the presumed remains is below the film limit. We deduce from the known geometry of the trajectory and the sensitivity of the optical system that at this altitude, $M>-1$ (see Figure 3); i. e., the intensity is less than that produced by about $30 \mathrm{~kg}$ of Lost City by a factor of more than $10^{3}$ ! Certainly a $1-\mathrm{kg}$ meteoritic stone would have been easily observable under these conditions, and indeed we would be surprised if $10 \mathrm{~g}$ could go undetected. For whatever reas on, this meteoroid essentially disappeared during the terminal flare, and its terminal mass, in the usual sense of finite fragments, is trivial at best.

B. Comparis on of Photometric and Dynamic Masses

In the upper curve of Figure 6, we show a comparison of the sum of the estimated terminal mass, $25 \mathrm{~kg}$, and photometric mass with the dynamic mass derived from the values of $v$ given in Figure $4 \underline{a}$. We have chosen $C_{D}=1.2, A=2.0$, and $\rho_{m}=3.73 \mathrm{~g} / \mathrm{cm}^{3}$ for the portion of the trajectory after fragmentation. The latter two values are, respectively, the measured shape factor of the main mass when it presents a maximum area to the flight direction, and the bulk density of the meteorite as determined by Clark et al. (this issue). The value of $C_{D}$ is that deduced from the trajectory data and the measured shape factor (Section 3). The luminous efficiency factor used was that described in Section 4B. The agreement for this portion of the curve is far better than the data demand. The prefragmentation trajectory data have been fitted to the photometric mass at $t=6 \mathrm{sec}$ with a value of $C_{D} A=3.80$. The poor agreement may well be attributed to the weak determination of $v$ for this interval.

The ratio of the initial mass to the terminal mass is, for the assumptions used here, 480/25. There is considerable evidence from measurements of cosmic-ray tracks and from studies of radio isotopes produced by cosmic 
rays that many meteorites do not ablate such a large fraction of theix mass. If Lost City ablated only 4 or $5 \mathrm{~cm}$ on all surfaces, its initial mass would have been roughly $50-100 \mathrm{~kg}$, depending on the actual end mass. We can decrease the photometric mass by applying an arbitrary linear correction factor, $k$, to the luminous efficiency. The plotted mass curve for $k=8$ is also shown in Figure 6. In this case, we have used the minimum terminal mass corresponding to the $17 \mathrm{~kg}$ of collected material to improve the fit with the dynamic mass. Even so, the agreement with the $m_{d}$ curve for $C_{D} A=1.7$ is quite poor.

Although the true correction to the provisional photometric mass is unlikely to be the simple scale factor $k$, no other variation can force a fit between the two masses so long as the initial and final masses are so constrained. We conclude, then, that either the correction to the provisional $m_{p}$ is very much less than $k=8$ or that $C_{D} A$ is not a constant during the last portion of the trajectory. For example, if $C_{D} A$ increases linearly with time and takes on values of 1.27 (about that for a sphere) at $t=6.5$ and 2.38 at $t=8.75$, the dashed curve labeled $C_{D} A=f(t)$ in Figure 6 would result. It is readily shown by application of equations (6) and (7) that this model (together with $C_{D}=$ constant) predicts that the frontal area of the body is unchanged during an ablation process that has reduced the mass to about one-third. This behavior may be unlikely, but it is not impossible. However, if the terminal mass proves to be substantially greater than the present recovered mass, the model could be satisfied only by an increase in area occurring while even less mass was ablated. The se conditions are met only if the body changes orientation during the last portion of the trajectory. The main mass shows every indication of having had a very stable flight for some period of time. We believe, therefore, that $1<k<8$ are probable extreme limits to the correction to our luminous efficiency. If time variations of $A$ are present and important, further refinements to the correction are not possible from our data alone. We require, at the least, an upper limit of the initial mass.

The results of a similar analysis for 40617 are shown in Figure 7 . The bulk density of Lost City was assumed for this object. It is possible to fit $m$ with a wide range of values of $k$ when the terminal mass is taken as a 
free parameter. It is notable, though, that if our best estimate of the ter minal mass, $10 \mathrm{~kg}$, is used, then $\mathrm{k}=1$ again gives the best fit. If $\mathrm{k}=8$ and $\mathrm{C}_{\mathrm{D}} \mathrm{A}=1.13$ throughout the postfragmentation trajectory, the three terminal fragments of $1 / 3 \mathrm{~kg}$ each would be approximately spherical and would have presentation areas of about $25 \mathrm{~cm}^{2}$, or about $7 \%$ of that of Lost City. It is unlikely that this body would produce $10 \%$ of the intensity of Lost City in an atmosphere of one-third the density. Values of $k=4$ and $m_{t}=3 \mathrm{~kg}$ (Figure 7) cannot be excluded by these arguments.

\section{INFLUX RATE AND BULK DENSITY OF METEOROIDS}

Lost City was the first meteorite recovery in 6 years of operation of the Prairie Network. When the program began, we expected about one fall of $1 \mathrm{~kg}$ or larger per year, based on Brown's [1960] statistics. We also expected to learn much from the first success. The latter expectation has been justified, but our results cause us to wonder whether it has been our analysis of the data or a paucity of falls that is primarily responsible for our previous lack of success.

We here summarize the Lost City results and our other search efforts to give some answers to this question and to the more general question of the structure or densities of average fireballs.

We planned to initiate a search when the observations were good and when the terminal ballistic coefficient was $v \gtrsim 20 \mathrm{~g} / \mathrm{cm}^{2}$. The limit is given both because small bodies are difficult to find and because uncertainties in the wind correction increase with diminishing size. We have made exceptions to our rules in several cases. Searches were made for a poorly observed object that we believed to be large and for two well-observed small bodies falling through moderate wind fields. Extensive search efforts were made for six events (the Networkstaff has walked about $5,000 \mathrm{mi}$ ). We began four of these searches, including Lost City, with some confidence of success. Two of the meteorites had terminal ballistic coefficients comparable to that of Lost City. Limited attempts were made for the recovery of five other 
suspected falls. Three of these had $v<20 \mathrm{~g} / \mathrm{cm}$. The other two were accompanied by sonic booms and gave other indications of a sizable fall. Unfortunately, clouds had obscured the primary cameras. Impact uncertainties were many kilometers.

In the past, we have commented on the small number of objects with sizable terminal ballistic coefficients and, either directly or by implication, have stated that this demonstrated our original estimates to be in error. In reaching this conclusion, we assumed an average spherical shape for meteorites. However, Lost City no. 1 proved to be about eight times the mass of an aerodynamically similar sphere. If this large correction factor were to be applied to all our other estimated terminal masses, we would conclude that our original fall rate was in fact underestimated. We would willingly admit complete uncertainty on the subject now if Lost City had not proved to be unique in several ways. First, we suspect that it is no coincidence that our deepest penetrating meteoroid produced the first recovery. Perhaps our other searches had been for bodies nearly as small as we estimated. Second, we remain puzzled by the ease of recovery of Lost City when compared to our more extensive searches that ended in failure. We acknowledge that outside assistance was partially responsible for fragments 2 and 3 (and apparently the assistance of Providence for fragment 1). But we believe a recovery by local residents is more likely in farmland, where most of our attempts were made, than in the range or woodland of Lost City. Furthermore, we believe we had at least $75 \%$ chance of recovering no. I had it not been on the road. We are certain that no. 4 would have been recovered within a week or two of the beginning of the search. Again we suspect that the end mass of Lost City was larger than for our other events and that recovery efforts were successful because there was more material to be found.

The flux of large bodies of meteoritic material entering the atmosphere has been another quantity of interest. Our preliminary estimates on this [McCrosky, 1968a] were based on photometric masses using our best estimate for the luminosity law applicable to smaller bodies. These estimated fluxes were higher than would be predicted by simple extrapolation of the small-body data but were reasonably consistent with airwave observations reported by 
Shoemaker. The reader is referred to a review by Gault [1970] for further details.

A large input mass together with the surprisingly low terminal mass suggested that the average meteorite underwent nearly total ablation. This conclusion was inconsistent with the modest ablation rates found from shielding determined from various cosmic-ray effects in meteorites. We concluded that most fireballs were not produced by meteorites.

Estimates of the bulk density of meteoroids have been made by considering this quantity the major unknown in equation (7). The governing relationship is

$$
\rho_{m}^{2}=\frac{\left(C_{D} A v\right)^{3}}{m_{p}+m_{t}}
$$

McCrosky and Ceplecha [1970] found that most Prairie Net meteors had bulk densities between 0.1 and $1.5 \mathrm{~g} / \mathrm{cm}^{3}$, with an average value of about $0.5 \mathrm{~g} / \mathrm{cm}^{3}$. This was not an entirely independent confirmation of the nature of the meteoroid. If we were overestimating the initial masses by using an erroneous luminosity law and at the same time underestimating dynamic (and terminal) masses because the average shapes were flatter than assumed, we would, by equation (14), be attributing too low a density to the material.

McCrosky and Ceplecha assumed spherical shapes $\left(A=1.21, C_{D}=0.92\right)$ and a constant luminosity coefficient $\tau_{0}=10^{-19} \mathrm{cgs}$. The new luminosity law used in the present analysis cannot be expressed accurately in the simple form, but it can be reasonably well approximated by $\tau_{0}=2.5 \times 10^{-19} \mathrm{cgs}$.

If we now assume that the average fireball obeys the same luminosity law as does Lost City for $k=1$ and that this average object also has initially the flattened shape represented by $C_{D} A=2.4$, then densities were previously underestimated by a factor of $(2.4 / 1.11)^{3 / 2} \times(2.5)^{1 / 2} \approx 5$. Comparable corrections are indicated by the data on 40617 after fragmentation. If, however, 
the average meteoroid has the flattening represented by the value of $C_{D} A=3.39$ for 40617 before fragmentation, then our earlier estimates would be in error by a factor of 8 . Essentially the same result obtains if we take $k>1$ and use corresponding lower values of $C_{D} A$. Stated otherwise, had we not known the bulk density and shape of Lost City, we would have concluded that it was a low-density meteoroid.

The excellent agreement between the two mass scales for $k=1$ (Section 5B) would appear to place the burden of the correction on the shape factor rather than on the luminous efficiency. If we are to attribute the small ratio, mass/area, of all fireballs to the shape factor, rather than to density, we must also be prepared to specify (a) the means of production of flat meteoroids, and (b) why they should, almost universally, traverse most of their trajectory in a maximum-drag orientation. If (a) is possible, then any body for which (b) fails will appear to have an exceptionally high value of $\mathrm{m} / \mathrm{A}$. No more than a few percent of meteors appear in this category [McCrosky and Ceplecha, 1970$]$.

Condition (a) is satisfied eithe $r$ by the ad hoc proposal that flat objects are produced in the crushing of the meteorite parent body or by space etching of meteoroids of less extreme shape, a suggestion made and rejected by McCrosky and Ceplecha. With only one case in hand, we are not yet compelled to assume that flattening is a common characteristic of meteorites. We note, though, that this attribute would affect the interpretation of cosmogenic isotope data and should be considered for Lost City, where the evidence for an initial flat shape is rather good.

We have proceeded thus far on the premise that Lost City and 40617 are typical of all fireballs. But in fact, Lost City is unique in our collection of data. Only one other meteor may have penetrated a comparable amount of atmosphere, and we have never before seen meteor fragments persist for such a large fraction of the trajectory. Furthermore, we have an example in 40503 of a meteor with grossly different behavior that must, we believe, result from a very different material. We can test three hypotheses regarding 40503. 
(1) The meteor is in every way similar to other meteoritic stones. This statement is false since an object of some considerable size was observed to disintegrate totally under thermal or pressure stresses that other meteorites - and Lost City in particular - withstood with only minor disruption.

(2) The meteorite is identical to ordinary meteorites in its chemistry and in its bulk properties except for strength. The mass-luminosity law that appears adequate for understanding Lost City $(k=1)$ is also applicable to this body. If so, $\mathrm{m}_{\mathrm{p}}=16.5$ tons at $\mathrm{t}=4.75 \mathrm{sec}$ where we have determined $v=66 \mathrm{~g} / \mathrm{cm}^{2}$. We would conclude, then, that $C_{D} A=9.2$. If $C_{D}=1.78(\mathrm{a}$ maximum value for hypersonic flow), then $A=5.2$, corresponding to a disk with an unlikely diameter-to-thickness ratio of 13.

As noted earlier, the measurements of $\mathrm{m}_{\mathrm{p}}$ and $\mathrm{m}_{\mathrm{d}}$ are poor for this case. As probable extreme limits, we can assume that $m$ has been overestimated by a factor of 16 (an error of $3 \mathrm{mag}$ ) and $\mathrm{m}_{\mathrm{d}}$ underestimated by a factor of 2 ; then $C_{D} A=1.8$ and the initial mass is 1 ton. Half of this initial mass is lost during the bright flare of 0.1-sec duration. Suppose the flare is caused by a disruption into many small pieces, each of which then undergoes an independent ablation process that consumes the fragment during the period of the flare. The lifetime, $\Delta t$, of a meteoroid fragment of mass $\mathrm{m}_{\mathrm{i}}$ is comparable to the time required to encounter its own mass of atmosphere. For the present case,

$$
\mathrm{m}_{\mathrm{i}} \approx \mathrm{A} \rho \mathrm{V} \Delta \mathrm{t} \approx 10 \mathrm{~g}
$$

This model of a weak structure disintegrating into tens of thousands of fragments almost instantaneously is perhaps a possibility. However, if we greatly overestimated the errors in $m_{p}$, the mass dissipation in the flare is, to us, incredible.

(3) The meteoroid's extreme fragility results in its abrupt demise. The exceptional luminosity associated with the flare is derived from some physical process not accounted for in the classical meteor theory. The mechanical structure may be tentatively taken as that of a wealk carbonaceous chondrite of $p_{m}=2 \mathrm{~g} / \mathrm{cm}^{2}$. For a rough computation, let us further as sume that 
$C_{D} A=2$ and accept our value of $\nu=66 \mathrm{~g} / \mathrm{cm}^{2}$. These imply a mass of $m=0.6$ ton, or about $4 \%$ of that determined from the luminosity law; i. e., that law and the photometry contain a combined error of a factor of 25 . Much of the problem is resolved if the extreme exrors in photometry of $3 \mathrm{mag}$ are present in the proper sense. We doubt these large errors and believe that errors of about $2 \mathrm{mag}$ are more reasonable. This could reduce the mass discrepancy to about a factor of 4 or 5, close to that suggested by Allen and Baldwin [1967] for the correction to the luminous efficiency for meteoroids of high carbon content when observations are made with panchromatic emulsions. (Highly refractory soot particles are expected to transfer an appreciable fraction of their kinetic energy into detectable blackbody radiation.) This event, then, can be understood if all the following are true: (a) the body is exceptionally weak; (b) it is quite flat; (c) the luminosity law for the meteor departs appreciably from that of Lost City; and (d) the photometry has a large error in the proper sense.

If the photometry is without error, we are at a los s to explain this object in terms of any known material without calling on an additional energy source in the meteoroid or in the atmosphere.

With the Lost City data in hand, we would no longer insist that the overwhelming majority of our fireballs are unlike normal meteorites. Some fraction of these can be meteorites disguised by shapes similar to that of Lost City. On the other hand, we can find no way to force meteor 40503 into the mold of a high-density object. If the brightest fireball can be other than a common meteorite, we suppose that some smaller objects can be as well.

\section{ORBITS}

The orbits of the three meteors are given in Table 1 . We also list the

only other well-determined orbit of a meteorite, Príbram [Ceplecha, I961], and the orbits of four other meteors with unusual characteristics thought to be indicators of meteorites in flight [Halliday, 1960; Cook et al., 1963; Jacchia et al., 1967]. 
They axe similar to the great majority of fireball orbits in having low inclinations and aphelia within Jupiter's orbit. Of the seven objects thought to be meteoritic (we exclude 40503 from this category), five have perihelion. distances $q>0.92 \mathrm{au}$, the others being $q=0.76$ a $u$ and $q=0.79$ au. The median value for fireballs with semimajor axis a $<5$ au is $q=0.71$ au [McCrosky, 1968b]. Perihelion distances for small, low-inclination or bits have a strong inverse correlation with the entry velocity, $V_{\infty}$. Thus, for the meteorites Pribram and Lost City, a relatively large value of $q$ was a necessary condition for their survival during entry. The other objects were also low velocity. This may not be a general characteristic of high-density material, however. An observational selection effect may be responsible for the apparent correlation. Low-velocity objects can produce long-enduring meteors, and it is the se that can be best analyzed for trajectory data.

We also note that the entry velocities and orbits of Príbram and 40503 are not dissimilar, but their behavior in the atmosphere was totally different. We conclude that with the limited data now available, the orbital parameters alone are insufficient to distinguish among the different types of meteors.

Acknowledgment. This work was supported in part by grant NGR 09-015-004 from the National Aeronautics and Space Administration. 


\section{REFERENCES}

Allen, H. J., and B. S. Baldwin, Jr., Frothing as an explanation of the acceleration anomalies of cometary meteors, J. Geophys. Res. , 72, 3483-3496, 1967.

Ayers, W. G., R. E. McCrosky, and C.-Y. Shao, Photographic observations of 10 artificial meteors, SAO Spec. Rept. 317,40 pp., 1970.

Brown, H., The density and mass distribution of meteoritic bodies in the neighborhood of the earth's orbit, J. Geophys. Res., 65, 1679-1683, 1960 .

Ceplecha, Z., Multiple fall of Príbram meteorites photographed, Bull. Astron. Czech., 12, 21-47, 1961 .

Clark, R., E. Jarosewich, and J. Nelen, Lost City, Oklahoma meteorite: An introduction to its laboratory investigation, This issue.

Cook, A. F., L. G. Jacchia, and R. E. McCrosky, Luminous efficiency of iron and stone asteroidal meteors, Smithsonian Contrib. Astrophys. , 7, 209-220, 1963.

Gault, D., Saturation and equilibrium conditions for impact cratering on the lunar surface: Criteria and implications, Radio Sci., 5, 273-291, 1970 .

Halliday, I., The spectrum of an asteroidal meteor fragment, Astrophys. J., $132,482-485,1960$.

Jacchia, L. G., F. Verniani, and R. E. Briggs, An analysis of the atmospheric trajectories of 413 precisely reduced photographic meteors, Smithsonian Contrib. Astrophys., 10, 1-139, 1967.

McCrosky, R. E., The distribution of magnitudes, masses, and energies of large meteoric bodies, SAO Spec. Rept. 280, 13 pp, $1968 \mathrm{a}$.

McCrosky, R. E., Orbits of photographic meteors, in Physics and Dynamics of Meteors, edited by L. Kresák and P. M. Millman, I. A. U. Symp. 33, D. Reidel, 265-279, 1968 b.

McCrosky, R. E., The Lost City meteorite fall, Sky and Tele., 39, 2-6, 1970.

McCrosky, R. E. and Z. Ceplecha, Photographic networks for fireballs, in Meteorite Research, edited by P. M. Millman, 600-612, 1969. 
McCrosky, R. E. and Z. Ceplecha, Fireballs and the physical theory of meteors, Bull. Astron. Czech., 21, 271-296, 1970.

McCrosky, R. E. and A. Posen, Special data-reduction procedures for Prairie Network meteor photographs, SAO Spec. Rept. 273, 88 pp., 1968. 


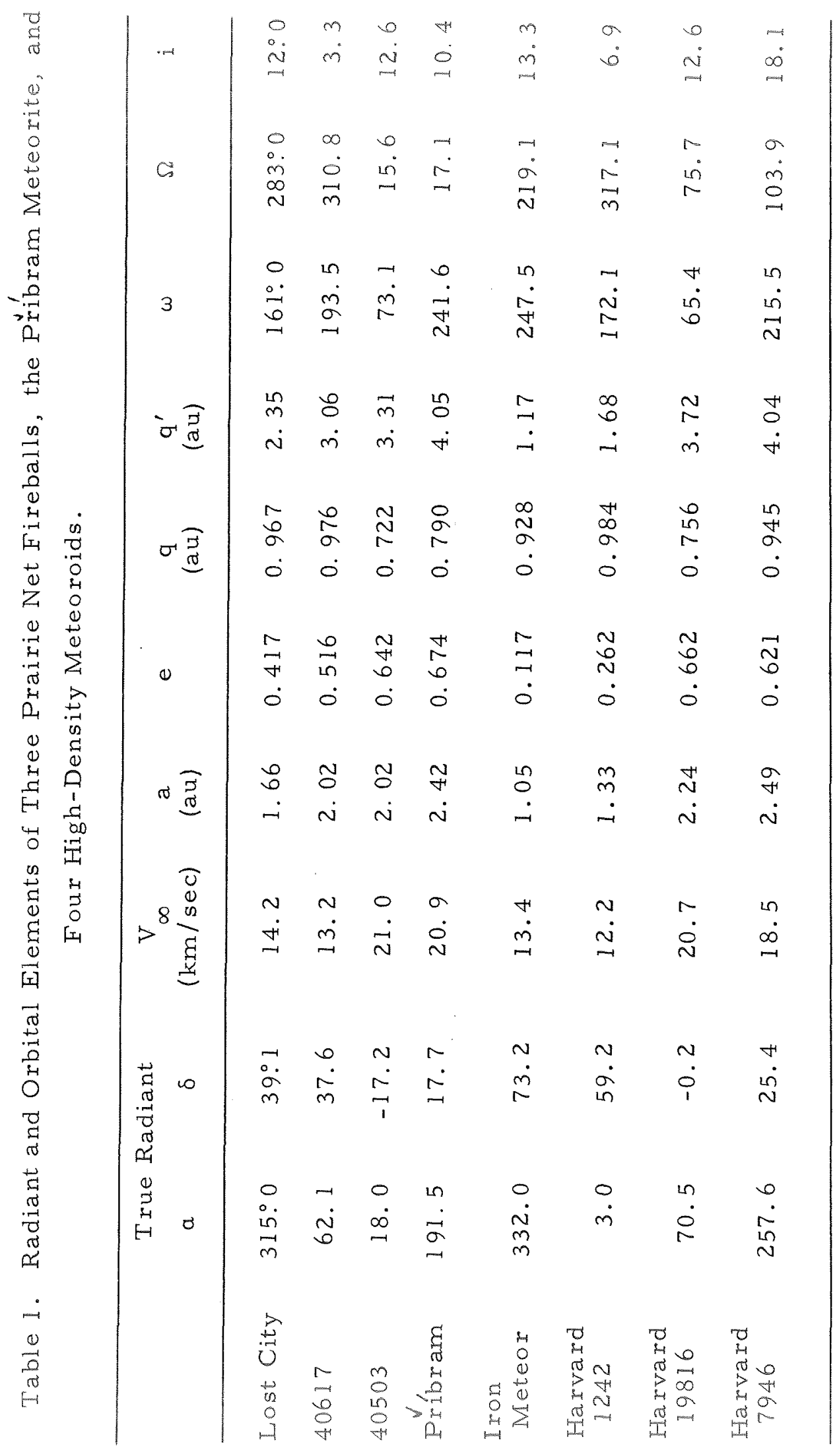



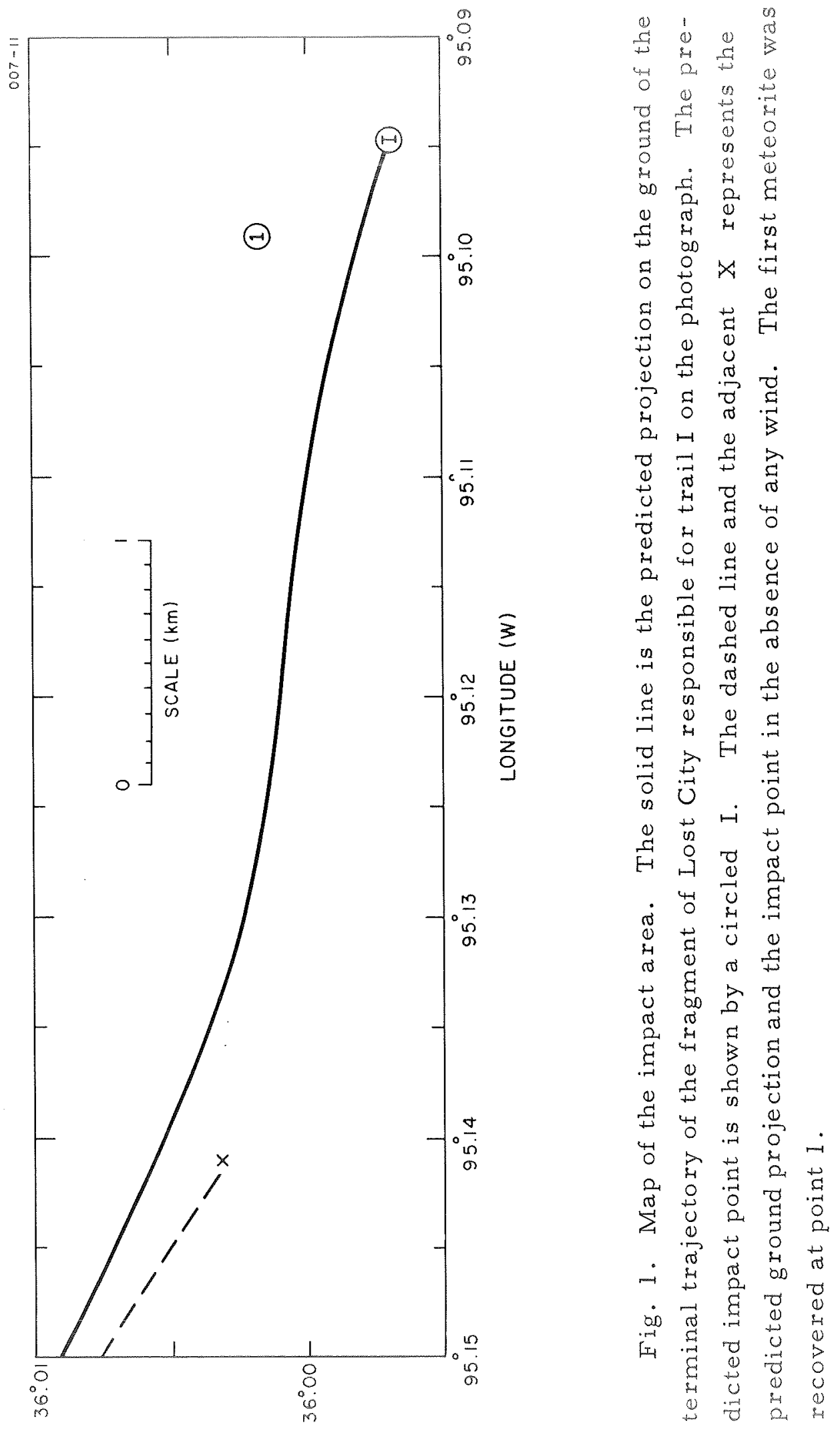

(N) $30 n 11167$ 


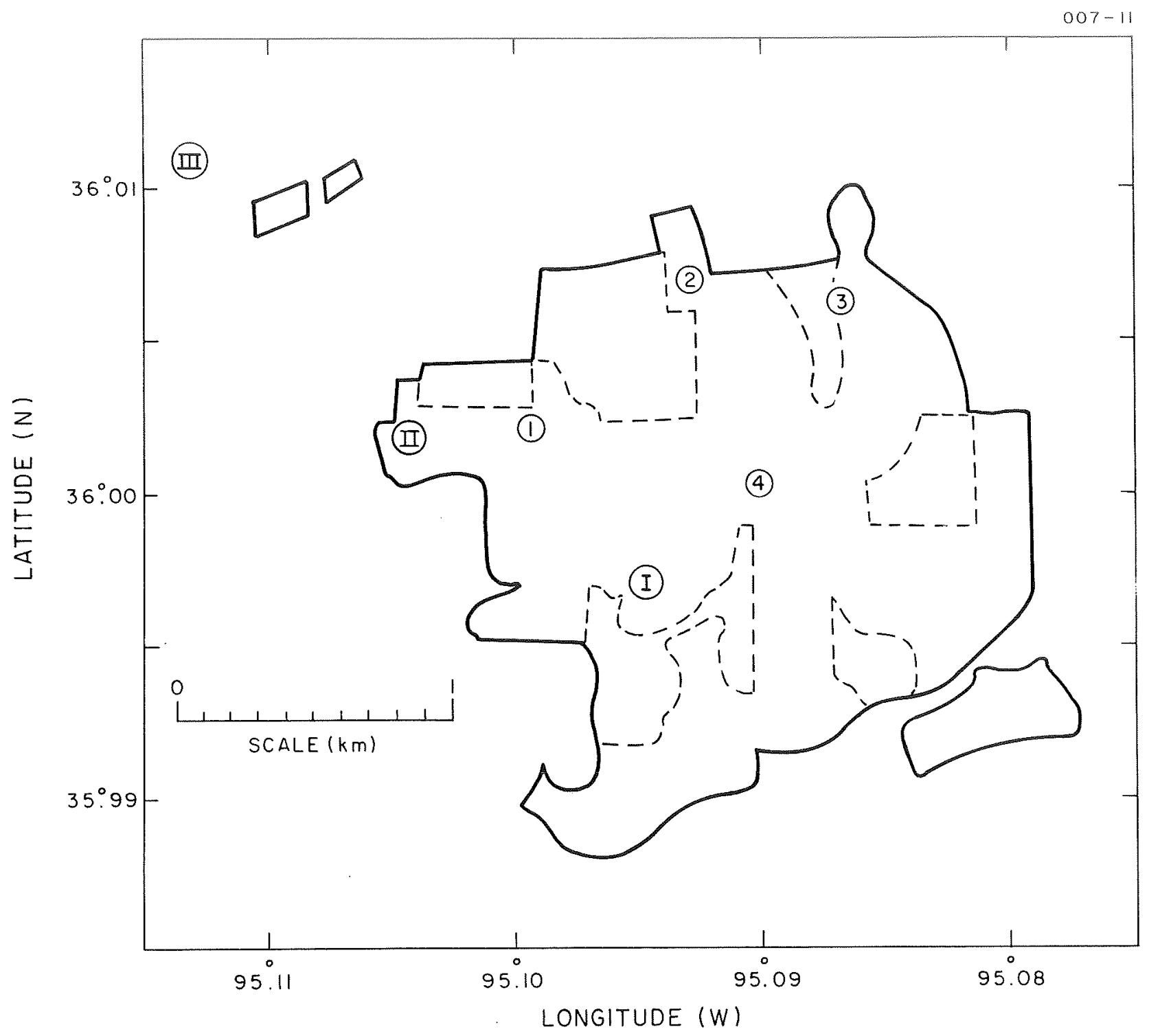

Fig. 2. Map of the meteorite search area. The Roman numerals mark the predicted impact points derived from the three trails photographed at the end of the trajectory. Points II and III were poorly determined. The Arabic numbers are positions of meteorite recoveries. The numbers are in chronological order of discovery as referred to in the text. The area searched by the Prairie Network team is shown by solid lines. The areas bounded by dashed lines are the more difficult forested regions that have been searched less thoroughly. 


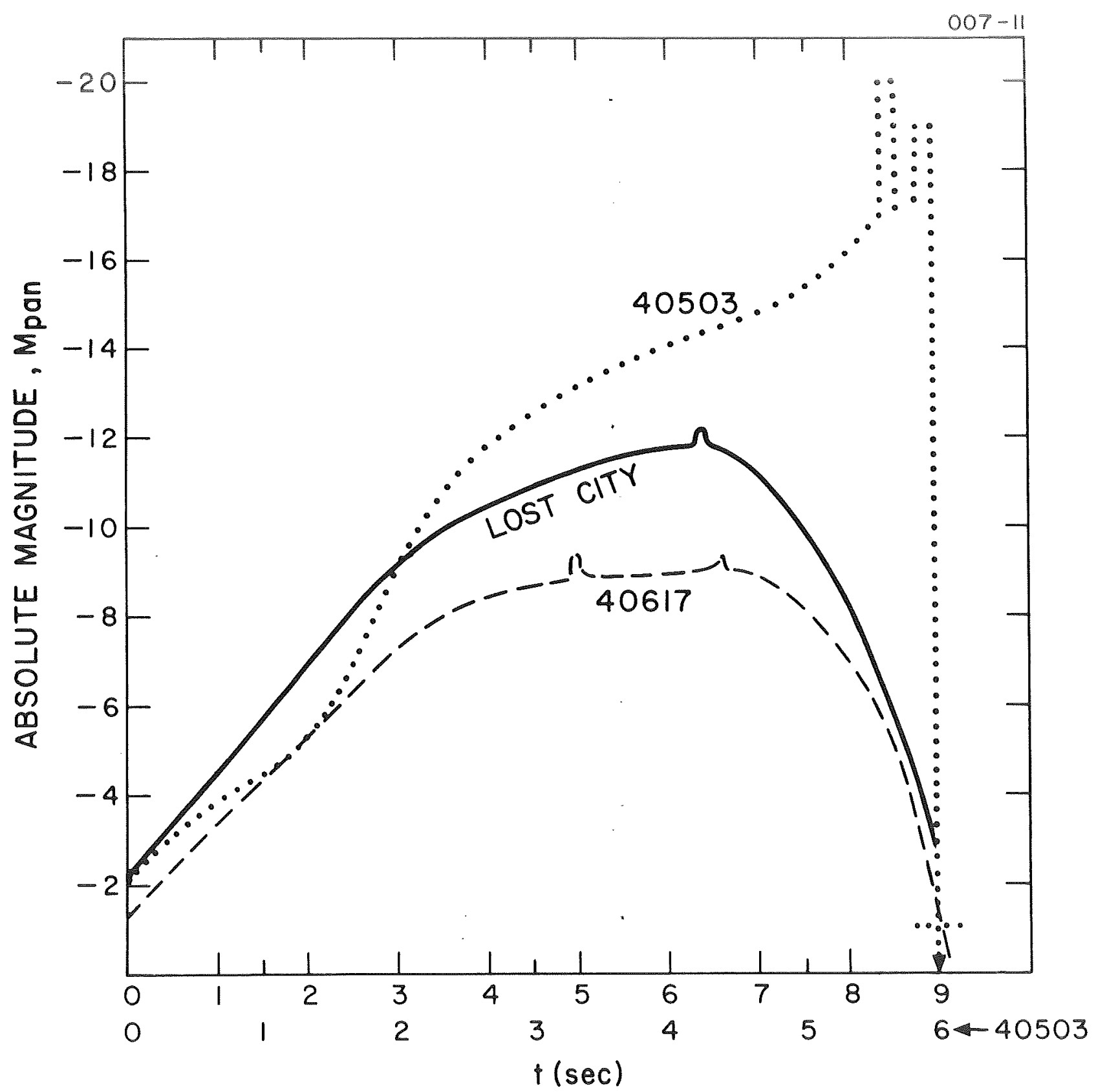

Fig. 3. Light curves of three fireballs. Note the expanded time scale for meteor 40503. The limit shown at $t=6$ "sec for this meteor is the limit of sensitivity of the photographic system. Times of flaxes on Lost City and 40617 are shown schematically. 


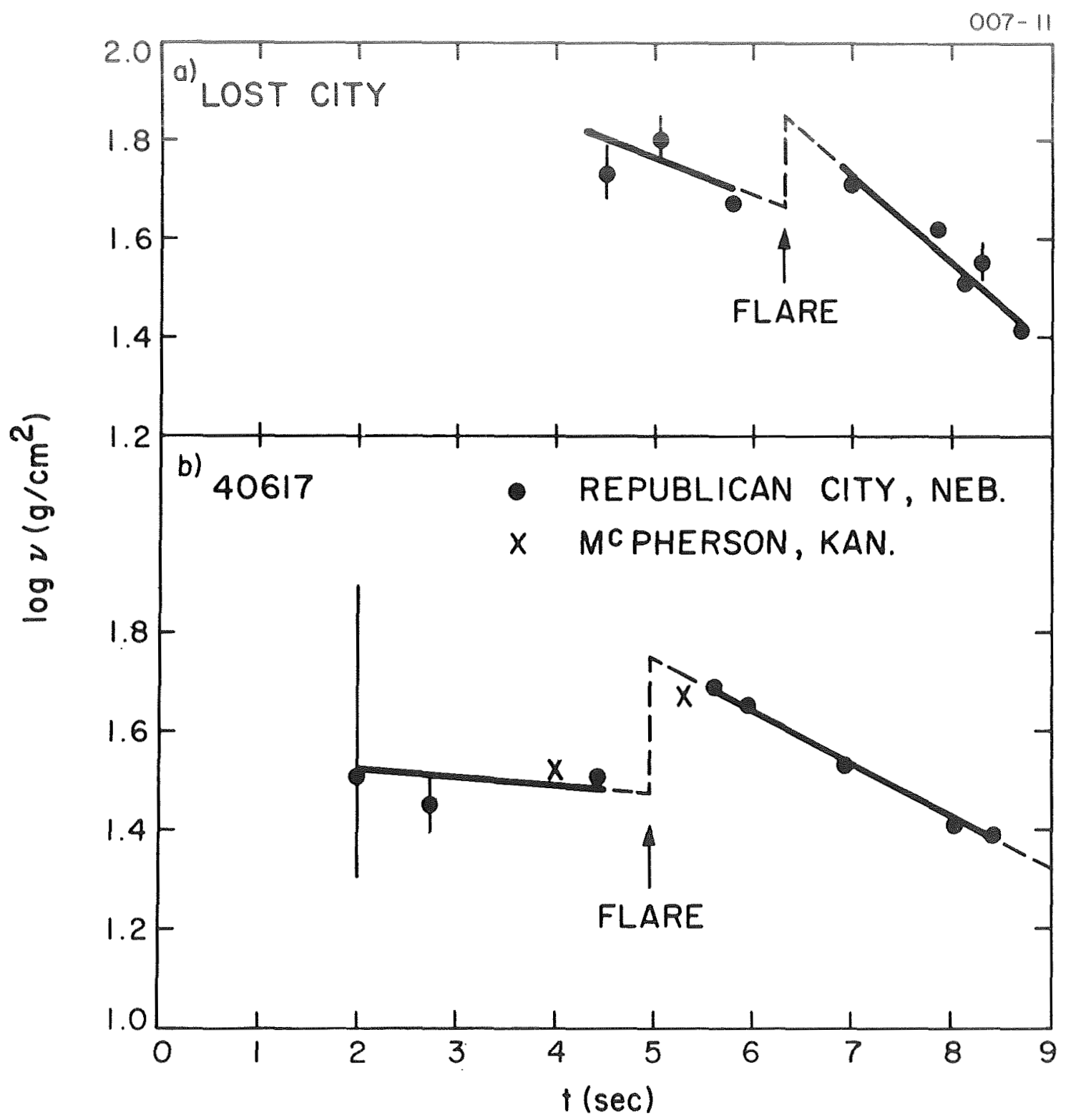

Fig. 4. Ballistic coefficients, $\nu$, of two fireballs. Measured values after the flares have been increased by 0.10 (a) and 0.16 (b) to adjust for the occurrence of multiple fragments. Dashed lines represent a possible behavior near the time of the meteor flares. RMS errors, when larger than the plotted points, are shown by vertical bars. 


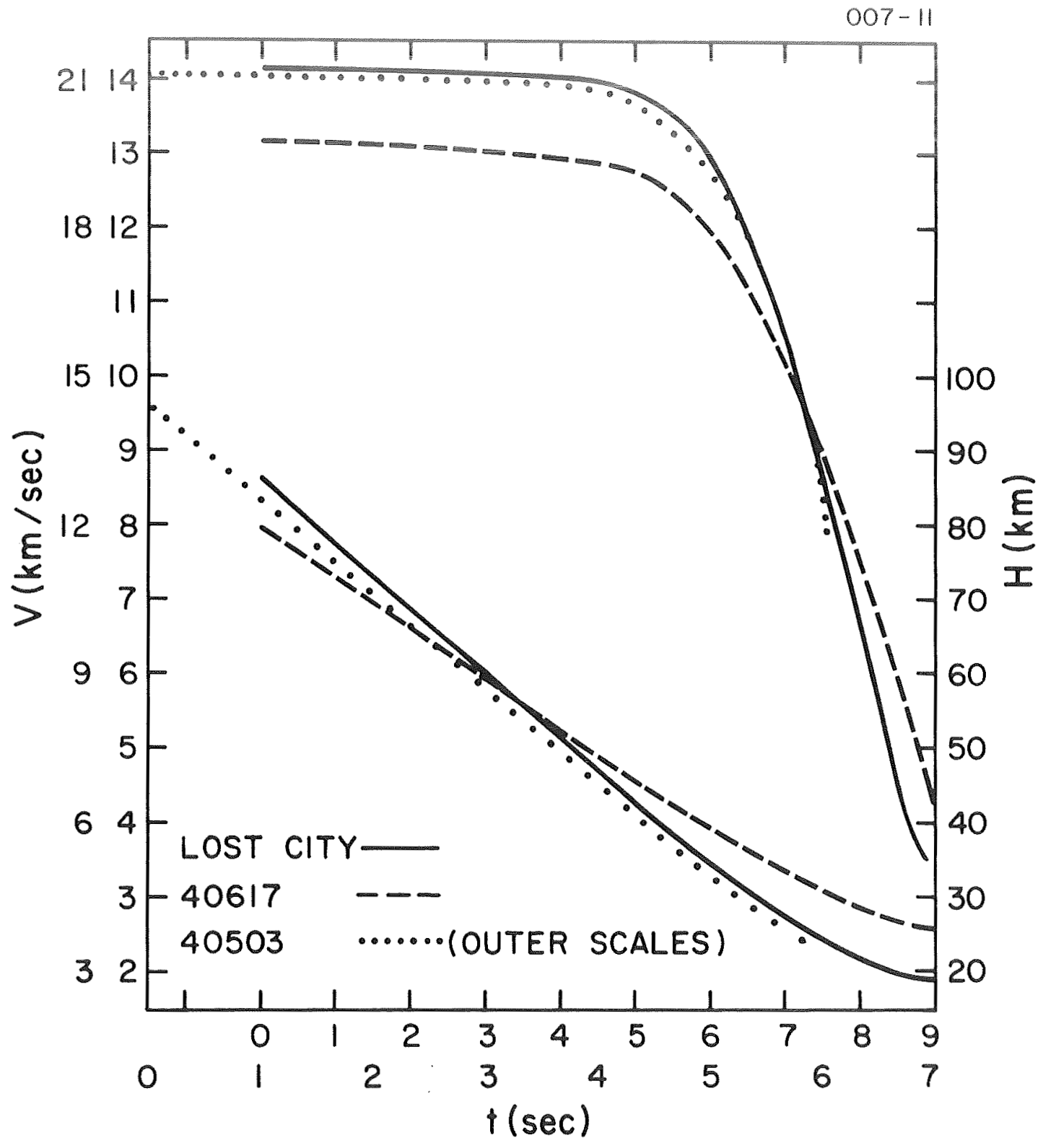

Fig. 5. Trajectory history of three fireballs. Height-time curves appear as the group of nearly straight lines in the lower left. The other group of curves are velocity-time plots. The outer scales of time and velocity apply only to meteor 40503. The time scale for meteor 40503 has been expanded by a factor of 1.5 relative to the other meteors (and the velocity scale contracted by a like factor) to exhibit a fortuitous similarity between this meteor and Lost City. 


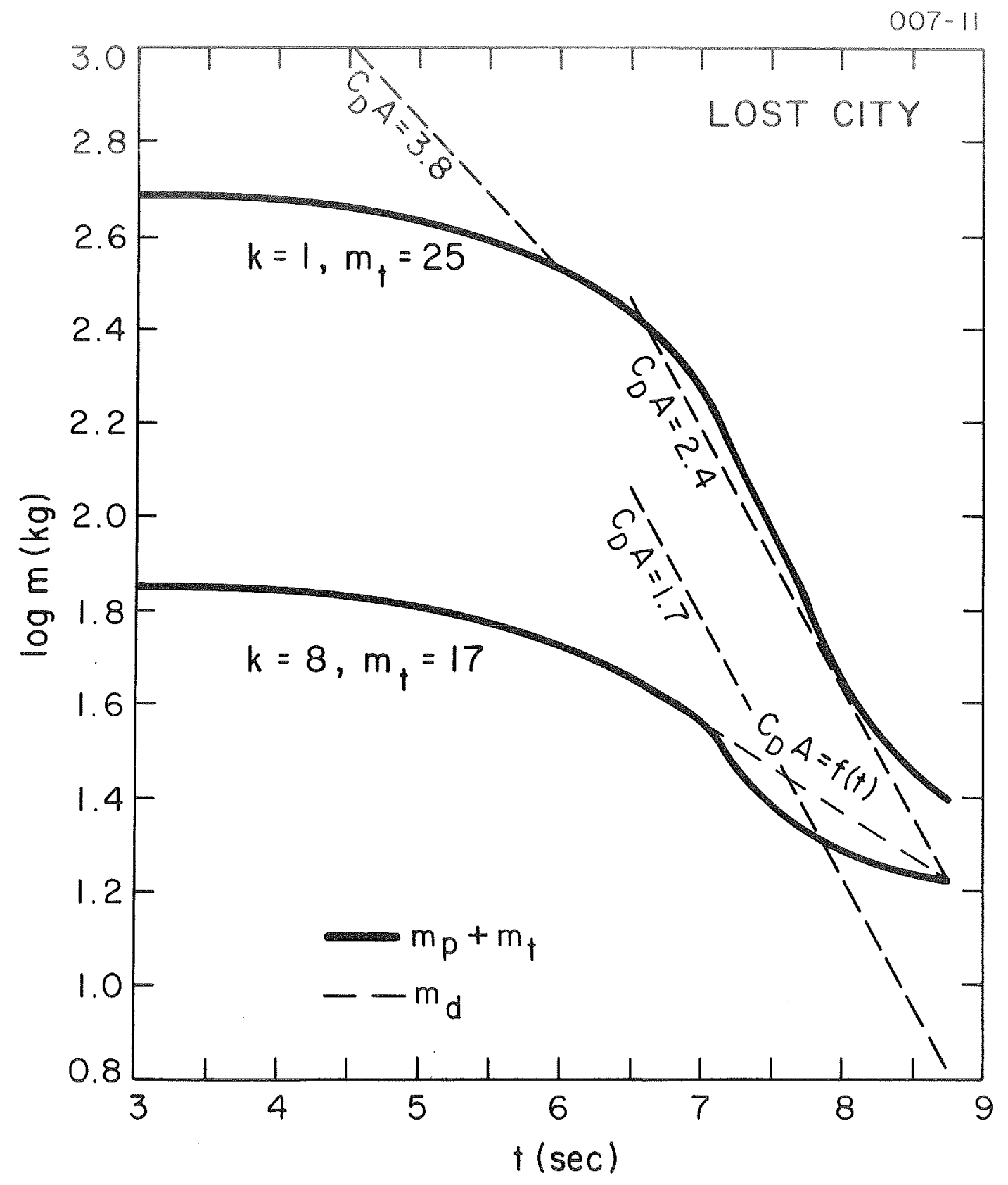

Fig. 6. Comparison of photometric mass $\left(m_{p}\right)$ plus terminal mass $\left(m_{t}\right)$ of Lost City with its dynamic mass $\left(m_{d}\right)$. The luminous efficiency law given in the text is varied by the factor $k$. The ballistic coefficients shown in Figure 4 a have been converted to dynamic masses by the choices of $C_{D} A$ shown in this figure. Preatmospheric masses derived from either model, $k=1$ or $k=8$, are essentially indistinguishable from the masses at $t=3 \mathrm{sec}$ and are, respectively, $490 \mathrm{~kg}$ and $72 \mathrm{~kg}$. 


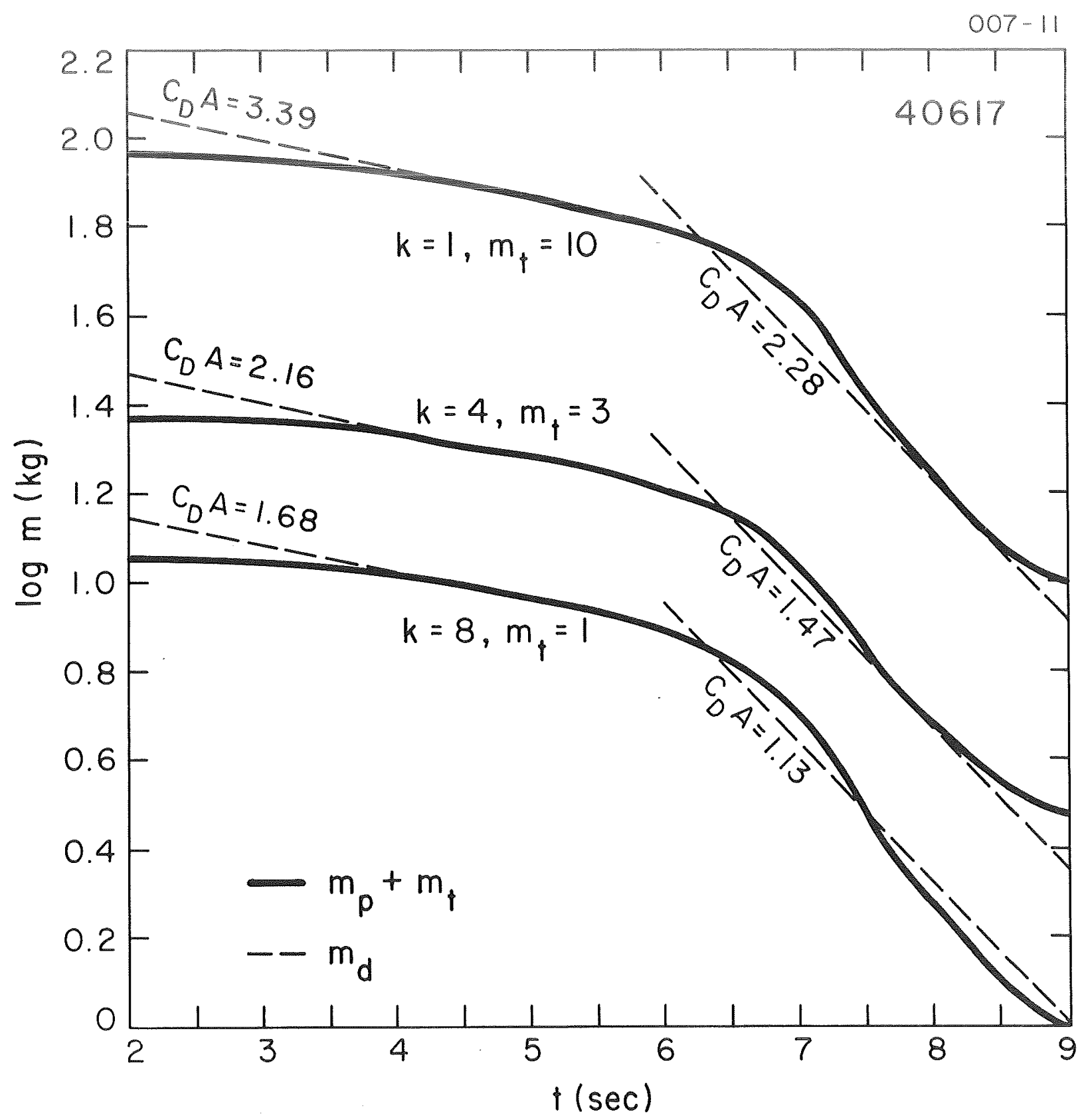

Fig. 7. Comparison of photometric mass $\left(m_{p}\right)$ plus terminal mass $\left(m_{t}\right)$ of meteor 40617 with dynamic mass $\left(m_{d}\right)$. The luminous efficiency law given in the text is varied by the factor $k$. The dynamic mass is derived from the ballistic coefficient shown in Figure $4 \mathrm{~b}$ and the values of $\mathrm{C}_{\mathrm{D}} \mathrm{A}$ given in this figure. Preatmospheric masses for the cases $k=1,4$, and 8 are $98 \mathrm{~kg}$, $23 \mathrm{~kg}$, and $11 \mathrm{~kg}$, respectively. 